\title{
Peripancreatic Tuberculosis With Involvement of The Porta Hepatis in a 41-year-old Thai Female Diagnosed via Fine NeEdle Aspiration
}

\author{
Paurush Shah, MD and Ali Siddiqui, MD
}

\section{Case Report}

A 41-year-old Thai female with past medical history of gastroesophageal reflux disease and duodenal ulcer who immigrated to the United States six years ago presented with a complaint of intermittent right upper quadrant (RUQ) pain for the last several years. Over the last year, she reported that the pain had significantly increased in intensity. She described the pain as crampy, beginning in the RUQ and radiating around to her back. There was no associated nausea, vomiting or weight loss. The patient had initially presented to an outside hospital a few months prior, was diagnosed with costochondritis, and was subsequently discharged. Her family promptly drove her to the emergency room of the outside hospital with severe abdominal pain and fevers. A computed tomography (CT) scan done in the emergency room showed a $4.6 \mathrm{~cm}$ x $2.8 \mathrm{~cm} \mathrm{x} 4.9 \mathrm{~cm}$ multiloculated mass adjacent to the head of the pancreas along with several enlarged lymph nodes in close proximity to the mass. The patient was referred to an oncologist at a specialty cancer center where an endoscopic ultrasound (EUS) was performed. The EUS revealed a $5.1 \mathrm{~cm}$ x $2.2 \mathrm{~cm}$ hypoechoic, heterogeneous, septated lesion. Fine needle aspiration (FNA) was performed on the mass and cytology was sent to determine the etiology of the peripancreatic mass. The EUS-FNA cytology demonstrated necrotizing granulomatous inflammation, but specials stains for tuberculosis and fungal etiologies were negative. Peripancreatic fluid, porta hepatis fluid and sputum cultures at this time were all negative.

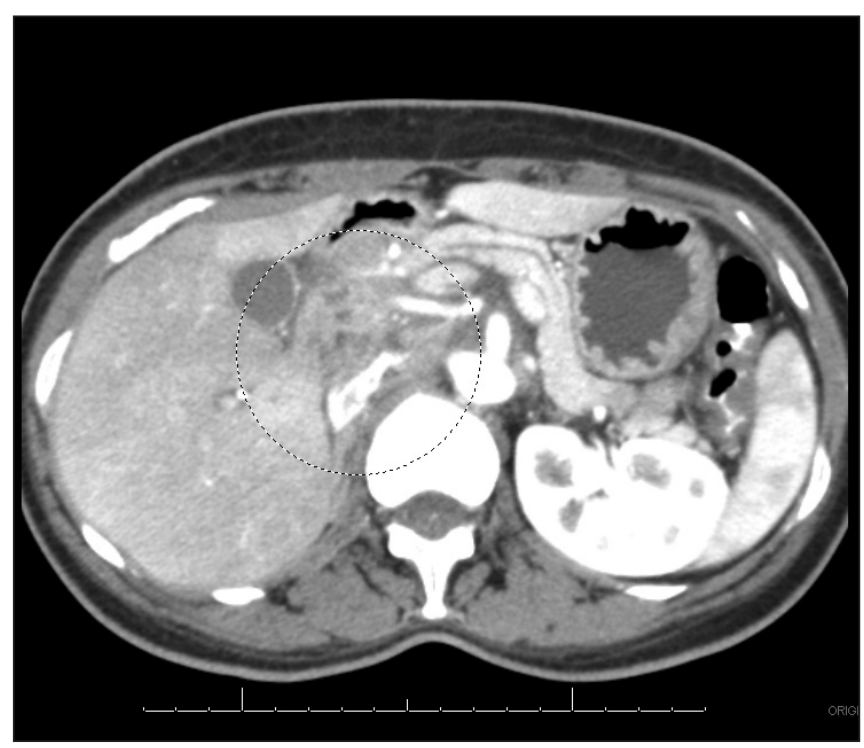

Figure 1. Abdominal CT showing a multiloculated cystic mass in the porta hepatis, portacaval, and peripancreatic regions.
On admission, the patient's vital signs were stable. She was alert, awake, and oriented. She had a normal temperature on admission but became febrile to $102 \mathrm{~F}$ in the emergency room. Her heart and lung exam were within normal limits. On abdominal exam, she was found to have significant RUQ and epigastric tenderness, with no distention, rebound, or guarding. She had normally active bowel sounds with no palpable organomegaly. At presentation, her white blood cell count was slightly elevated at $11.1 \times 10^{3} / \mu \mathrm{L}$ but the rest of her complete blood count panel and basic metabolic panel were within normal limits. Her amylase was $85 \mathrm{IU} / \mathrm{L}$, and her lipase was $20 \mathrm{IU} / \mathrm{L}$. Her hepatic function panel was within normal limits except for a slightly elevated alkaline phosphatase level at $108 \mathrm{IU} / \mathrm{L}$.

After being transferred to Thomas Jefferson University Hospital, a chest CT and repeat abdominal CT and EUS were performed. The $\mathrm{CT}$ of the abdomen showed a multiloculated cystic mass in the porta hepatis, portacaval, and peripancreatic region measuring approximately $4.3 \mathrm{~cm} \times 3.1 \mathrm{~cm}$ (Figure 1). Additionally, severe narrowing of the distal main portal vein and narrowing of the left portal vein from the adjacent mass were described. The porta hepatis component of the mass had slightly increased in size and demonstrated increased cystic changes and increased compression of the main portal vein and left portal vein in comparison to a $\mathrm{CT}$ done a month ago. The pancreas itself was not involved and appeared unremarkable, but had been displaced by the mass. No pancreatic ductal dilatation was observed.

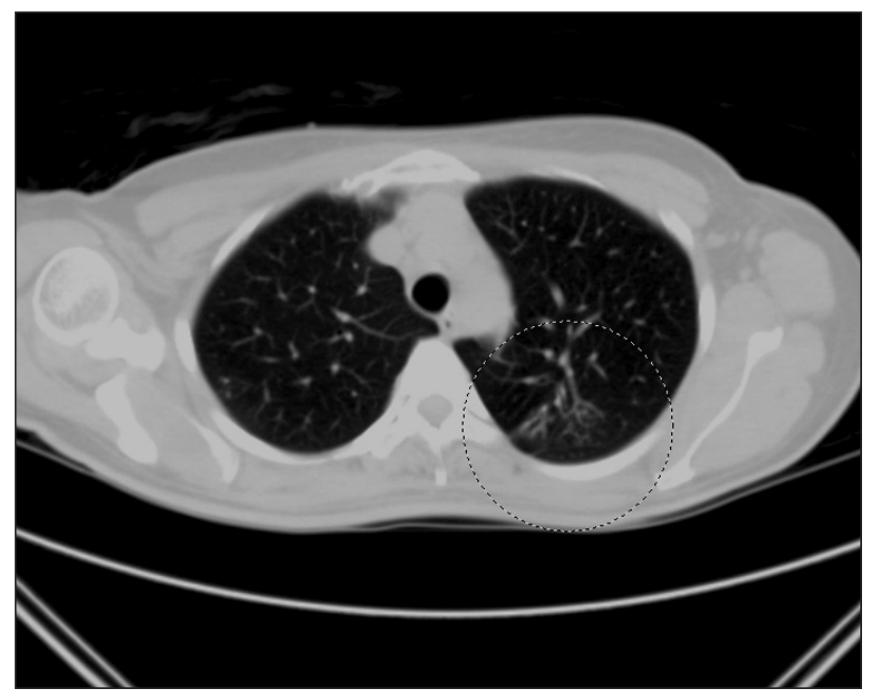

Figure 2. CT chest demonstrating bilateral upper lobe tree-in-bud opacities suggestive of infectious or inflammatory processes and endobronchial spread of infection. 


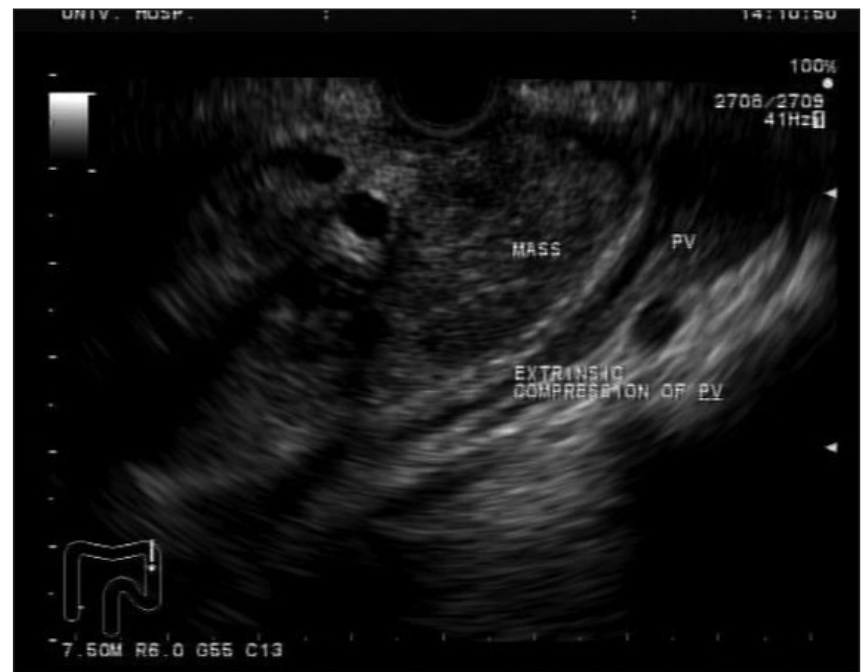

Figure 3. EUS illustrating a hypoechoic, lobular, homogeneous mass lesion present adjacent to the pancreatic head and compressing the portal vein.

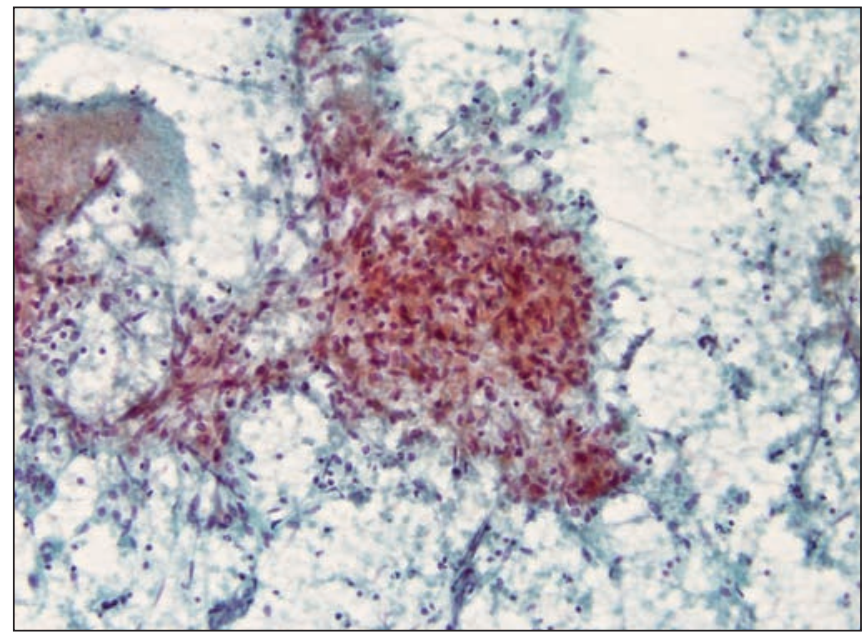

Figure 4. Cytology from aspiration of porta hepatis and peripancreatic masses showing a collection of epithelial histiocytes in the background of inflammatory cells and debris

The chest CT demonstrated bilateral upper-lobe "tree-in-bud" opacities suggestive of infectious or inflammatory processes including endobronchial spread of infection, such as seen with tuberculosis (TB) (Figure 2). The differential diagnosis also included other atypical mycobacterial organisms, viruses and fungal etiologies.

The repeat EUS described a $4.3 \mathrm{~cm}$ x $2.8 \mathrm{~cm}$ hypoechoic, lobular, homogeneous lesion present adjacent to the pancreatic head (Figure 3 ). The mass had a cystic component to it and compressed but did not invade the portal vein. The entire pancreas appeared normal with full visualization of the body

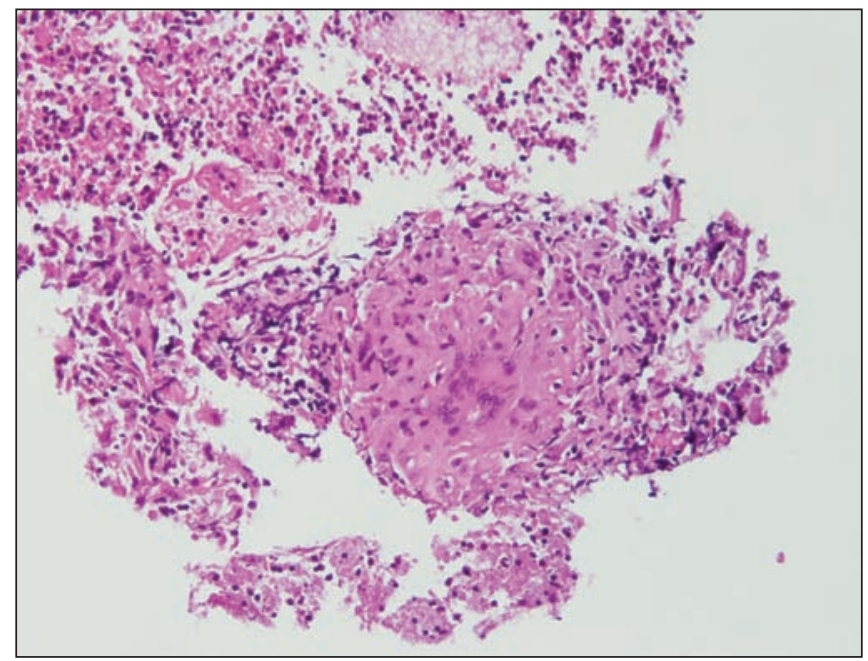

Figure 5. A cell block sample of the aspirate showing a classic granuloma with a multi-nucleated giant cell surrounded by epithelioid histiocytes in the background of lymphocytes and necrotic debris.

and tail. Several $1 \mathrm{~cm}$, oval, heterogeneous peri-pancreatic lymph nodes with poorly defined margins were also found. Examination of the fluid aspirated from the pancreatic lesions showed a collection of epithelioid histiocytes in a background of inflammatory cells and debris (Figure 4). Additionally, a cell block sample of the aspirate showed a classic granuloma with a multi-nucleated giant cell surrounded by epithelioid histiocytes in a background of lymphocytes and necrotic debris (Figure 5).

Staining of the fluid culture from the porta hepatis and peripancreatic masses were negative with specialty stains for acid-fast bacilli, but cultures eventually grew out mycobacterium tuberculosis (MTB) complex after 5 weeks. The state health department was notified. Follow up was arranged upon discharge for continuation of MTB treatment.

\section{Discussion}

MTB is an acid-fast bacillus that causes an infection known as tuberculosis. TB is characterized by chronic granulomas and had been a common disease of undeveloped countries decades ago, but has been well controlled with advancing antibiotic therapies more recently. Over the last few years however, there has been a rise in the incidence even in developed countries such as the United States due to increasing numbers of patients with immunocompromised states and due to increasing numbers of immigrants from areas with endemic TB. ${ }^{1,2,4,5,7} \mathrm{~TB}$ is a disease that can present almost anywhere in the body, but is most routinely discovered in the lungs. For example, TB of the digestive tract can occur anywhere from the esophagus to the anus. Additionally, tuberculosis can present as an abdominal infection, localizing in the liver, spleen, or ileocecal region resulting in non-specific findings such as fever, pain, malaise, 
weakness, weight loss, anorexia, and jaundice. ${ }^{1,2,-5,7}$ Pancreatic $\mathrm{TB}$, is exceptionally rare with only a handful of cases having been published. ${ }^{1,3,5}$ The reasoning behind this is unclear, but it is hypothesized that digestive pancreatic enzymes interfere with the seeding of mycobacterium into the pancreatic tissue. ${ }^{4,7}$ Pancreatic TB currently is being diagnosed more frequently, partly because of the increase in immunodeficient patients and partly due to improved pancreatic imaging.

Pancreatic TB is often indistinguishable from pancreatic tumors on CT imaging because both present as non-specific lesions in the pancreas; FNA with cytology is needed to make a definitive diagnosis. ${ }^{1,3,4}$ Positron emission tomography (PET) scanning is typically used to differentiate cancerous lesions from other concerning processes, but is not useful with TB related lesions, which can cause an increased uptake on PET scans, notably in the lungs. ${ }^{6}$ Low-attenuation peripancreatic and periportal adenopathy with peripheral rim enhancement may support a diagnosis of pancreatic TB on CT. ${ }^{2}$ In an article by Xia, in which characteristics of pancreatic TB were elicited via 16 patients and 58 prior literature reports, some associations and possible ways to distinguish pancreatic TB from pancreatic tumor were identified. Xia found that young people and females were more likely to have pancreatic TB, whereas pancreatic cancer was more commonly found in males and the elderly. Moreover, it was found that not all people had a past history of $\mathrm{TB}$, but most who develop pancreatic TB come from a region known to be endemic for TB. The most common symptoms that patients complained of were epigastric pain, fever, and weight loss, though these were not specific to pancreatic TB. ${ }^{1-5,7}$ Lastly, it was found that FNA with tissue cytology was the most definitive non-surgical way to diagnose pancreatic TB and rule out carcinoma. ${ }^{1,4-7}$

The spread of TB to the pancreas is poorly understood, but may occur as one of a number of different forms of infection. It may come from generalized, or military TB, in which case MTB is the most common causative agent. Spread to the pancreas can occur as a result of seeding from celiac, retroperitoneal, or peripancreatic lymph nodes. In this case, Mycobacterium bovis is likely the infectious agent. ${ }^{1,6,7}$ Also, spread to the pancreas may result from hematogenous dissemination of bacteria from the lungs. ${ }^{2,6,7}$ Lastly, a primary localized pancreatic TB may be seen. This is a very rare form of $\mathrm{TB}$, which points to the intestinal tract as the root of infection. ${ }^{1,6}$

\section{Conclusion}

Pancreatic TB is a rare but serious condition. The variability in imaging of pancreatic TB poses potential diagnostic problems. Pancreatic TB is curable with standard antibiotics if it is diagnosed early enough, but delay of diagnosis or misdiagnosis can potentially be fatal. ${ }^{2,3}$ In immunocompromised patients or young patients from areas endemic with TB in whom TB is clinically suspected, EUS-FNA should be performed in order to determine the etiology of the pancreatic lesion. ${ }^{4,5}$ The obtained sample should be sent for an acid-fast bacilli stain, as a preliminary test for mycobacterium species, even though the yield is low. Cultures should also be sent, though they can take up to six to eight weeks grow for definitive rule out. ${ }^{5}$ Patients should be started on antibiotics as soon as TB is clinically suspected. Often, they do very well with few lasting complications once treatment begins.

\section{References}

1. Xia F, Poon RT, Wang SG, Bie P, Huang XQ, Dong JH. Tuberculosis of pancreas and peripancreatic lymph nodes in immunocompetent patients: experience from China. World J Gastroenterol 2003;9:1361-4.

2. Pombo F, Diaz Candamio MJ, Rodriguez E, Pombo S. Pancreatic tuberculosis: CT findings. Abdom Imaging 1998;23:394-7.

3. Rezeig MA, Fashir BM, Al-Suhaibani H, Al-Fadda M, Amin T, Eisa H. Pancreatic tuberculosis mimicking pancreatic carcinoma: four case reports and review of the literature. Dig Dis Sci 1998;43:329-31.

4. D’Cruz S, Sachdev A, Kaur L, Handa U, Bhalla A, Lehl SS. Fine needle aspiration diagnosis of isolated pancreatic tuberculosis. A case report and review of literature. JOP 2003;4:158-62.

5. Riaz AA, Singh A, Robshaw P, Isla AM. Tuberculosis of the pancreas diagnosed with needle aspiration. Scand J Infect Dis 2002;34:303-4.

6. Sanabe N, Ikematsu Y, Nishiwaki Y, et al. Pancreatic tuberculosis. J Hepatobiliary Pancreat Surg 2002;9:515-8.

7. Kaushik N, Schoedel K, McGrath K. Isolated pancreatic tuberculosis diagnosed by endoscopic ultrasound-guided fine needle aspiration: a case report. JOP 2006;7:205-10. 